\title{
BMJ Global Health How to design a complex behaviour change intervention: experiences from a nutrition-sensitive agriculture trial in rural India
}

\author{
Helen Harris-Fry (D) , ${ }^{1}$ Meghan O'Hearn, ${ }^{2}$ Ronali Pradhan, ${ }^{3}$ Sneha Krishnan, ${ }^{1}$ \\ Nirmala Nair, ${ }^{4}$ Suchitra Rath, ${ }^{4}$ Shibanand Rath, ${ }^{4}$ Peggy Koniz-Booher, ${ }^{5}$ \\ Heather Danton, ${ }^{5}$ Ashley Aakesson, ${ }^{5}$ Shibananth Pradhan, ${ }^{6}$ Naba Kishore Mishra, ${ }^{6}$ \\ Abhinav Kumar, ${ }^{3}$ Avinash Upadhay, ${ }^{3}$ Audrey Prost, ${ }^{7}$ Suneetha Kadiyala ${ }^{1}$
}

To cite: Harris-Fry $\mathrm{H}$, O'Hearn M, Pradhan R, et al. How to design a complex behaviour change intervention: experiences from a nutritionsensitive agriculture trial in rural India. BMJ Global Health 2020;5:e002384. doi:10.1136/ bmjgh-2020-002384

Handling editor Valery Ridde

- Additional material is published online only. To view please visit the journal online (http://dx.doi.org/10.1136/ bmjgh-2020-002384).

Received 8 February 2020 Revised 10 April 2020 Accepted 13 April 2020

Check for updates

(c) Author(s) (or their employer(s)) 2020. Re-use permitted under CC BY. Published by BMJ.

For numbered affiliations see end of article.

Correspondence to Dr Helen Harris-Fry; helen.harris-fry@Ishtm.ac.uk

\section{ABSTRACT}

Many public health interventions aim to promote healthful behaviours, with varying degrees of success. With a lack of existing empirical evidence on the optimal number or combination of behaviours to promote to achieve a given health outcome, a key challenge in intervention design lies in deciding what behaviours to prioritise, and how best to promote them. We describe how key behaviours were selected and promoted within a multisectoral nutrition-sensitive agriculture intervention that aimed to address maternal and child undernutrition in rural India. First, we formulated a Theory of Change, which outlined our hypothesised impact pathways. To do this, we used the following inputs: existing conceptual frameworks, published empirical evidence, a feasibility study, formative research and the intervention team's local knowledge. Then, we selected specific behaviours to address within each impact pathway, based on our formative research, behaviour change models, local knowledge and community feedback. As the intervention progressed, we mapped each of the behaviours against our impact pathways and the transtheoretical model of behaviour change, to monitor the balance of behaviours across pathways and along stages of behaviour change. By collectively agreeing on definitions of complex concepts and hypothesised impact pathways, implementing partners were able to communicate clearly between each other and with intervention participants. Our intervention was iteratively informed by continuous review, by monitoring implementation against targets and by integrating community feedback. Impact and process evaluations will reveal whether these approaches are effective for improving maternal and child nutrition, and what the effects are on each hypothesised impact pathway.

\section{INTRODUCTION}

Many public health interventions aim to promote healthful behaviours, with varying degrees of success. ${ }^{12}$ Some interventions try to change behaviours by actively engaging participants using interactive techniques such
Summary box

Behaviour change interventions aiming to improve health outcomes can simultaneously focus on many, different behaviours

- To decide on which behaviours to promote in our nutrition-sensitive agriculture intervention, we used the following inputs: existing conceptual frameworks and behaviour change models, published empirical evidence, a feasibility study, formative research, the team's local knowledge and community feedback.

- As the intervention progressed, we mapped each of the prioritised behaviours against our hypothesised impact pathways and the transtheoretical model of behaviour change, to monitor the balance of behaviours across pathways and stages of behaviour change.

- Intervention design and implementation was aided by collective agreement between partners on definitions of complex concepts and hypothesised impact pathways, and the continuous review of implementation against targets and community feedback.

as interpersonal counselling, ${ }^{3}$ motivational interviewing, ${ }^{4}$ or Participatory Learning and Action (PLA) with groups. ${ }^{5-7}$ Other approaches can be less interactive but reach a wider audience, for example, through mass media $^{89}$ or text messaging. ${ }^{10}$

One challenge common to these interventions lies in deciding what behaviours to prioritise, and how best to change them, particularly when health outcomes are determined by several behaviours. For example, interventions aiming to reduce undernutrition could modify diets, physical activity, hygiene, or food purchasing and production decisions. ${ }^{11}$ Each of these could be divided into more specific behaviours, such as 'eat one additional meal during pregnancy' or 
'introduce complementary foods at six months of age'. It is easy to see how this could multiply, such that hundreds of behaviours could cumulatively improve a single health outcome. With increasing complexity of multisectoral interventions, such as nutrition-sensitive agricultural interventions, these options multiply even further and implementers have to make decisions regarding both agriculture and nutrition-specific impact pathways and behaviours. Implementers also have to decide how to adapt delivery platforms to address the multiple objectives of these complex interventions.

After selecting which behaviours to focus on, further analysis is required to identify how best to encourage their uptake. Enabling factors, which have been categorised by Michie, Van Stralen and West into people's 'capabilities', 'opportunities' and 'motivations' (the 'COM-B' model),${ }^{12}$ can vary in their relative importance across contexts, seasons and life stages. As articulated in the transtheoretical model, people may be at different stages in their process of adopting a behaviour-from thinking about it to trying it, and continuing with it. ${ }^{13}$

However, interventions cannot aim to change all relevant behaviours, or address all possible barriers. There are programmatic constraints on the number of activities an intervention can implement, and issues that an intervention can address, at an effective level of coverage. Moreover, addressing too many issues may be off-putting to participants-potentially causing information overload and choice fatigue, and inhibiting behaviour change. ${ }^{14} 15$

However, for health outcomes with a complex aetiology, we have limited understanding of how interventions should choose which behaviours to prioritise, or how many to promote. Tools such as the 'Behaviour Change Wheel' provide guidance on how to unpack the capabilities, opportunities and motivations that underlie a given behaviour. ${ }^{12} \mathrm{~A}$ few studies suggest that using more techniques to change behaviours increases intervention effectiveness, ${ }^{116}$ and we have some information on the relative effectiveness of varying doses and coverage. For example, increasing the number of participatory women's groups per population increases impacts on neonatal mortality, ${ }^{17}$ and larger effects are also observed with increasing proportion of pregnant women attending groups. ${ }^{18} \mathrm{~A}$ recent evaluation of a radio programme promoting vitamin A-rich sweet potatoes found that around 44 episodes were needed to improve knowledge. ${ }^{19}$

Although increasing dosage, coverage and numbers of techniques may increase intervention effectiveness, there is little guidance on whether an intervention should aim to change many behaviours, or focus on just a few, or on how barriers to behaviour change should be prioritised and addressed.

\section{A CASE STUDY FROM A NUTRITION-SENSITIVE AGRICULTURE TRIAL IN INDIA}

In this paper, we provide a case study of a complex nutrition-sensitive agriculture (NSA) intervention that aimed to reduce maternal and child undernutrition in rural India. Specifically, we describe how we (1) identified the six most important pathways through which we hypothesised our interventions would improve nutrition outcomes, (2) prioritised behaviours and barriers to behaviour change to address within these pathways, (3) reviewed our intervention content against these priorities and (4) continually integrated community participants' reported priorities.

The Upscaling Participatory Videos and Action for Agriculture and Nutrition (UPAVAN) trial is a four-arm cluster-randomised controlled trial aiming to improve maternal and child nutrition through the dissemination of locally developed videos, women's group meetings and follow-up home visits in rural Odisha, India. The primary outcomes are \% children aged 6-23 months consuming at least four food groups per day and maternal body mass index.

UPAVAN has seven partners. Digital Green coordinated intervention implementation; Voluntary Association for Rural Reconstruction and Appropriate Technology (VARRAT) implemented the interventions; JSI Research and Training Institute provided technical assistance on behaviour change, formative research and training; and Ekjut provided technical assistance on a PLA component. London School of Hygiene \& Tropical Medicine led all research activities, in collaboration with University College London and Development Corner Consulting.

Full details of the trial design are given in the protocol ${ }^{20}$ and an operational protocol detailing roles and responsibilities, intervention content, coverage, dosage, timings, monitoring systems and training plans is available in the online supplementary file 1.

\section{Study context, rationale and overview}

Rates of undernutrition in India are high: $38 \%$ of children under 5 years of age are chronically undernourished (height-for-age z-score $<-2 \mathrm{SD}$ ), a fifth are acutely undernourished (weight-for-height $z$-score $<-2 \mathrm{SD}$ ), and around a quarter of women are underweight (body mass index $\left.<18.5 \mathrm{~kg} / \mathrm{m}^{2}\right) .{ }^{21}$

Agriculture can play a role in improving nutrition outcomes. Programmes promoting home production of nutrient-rich foods (biofortification, homestead gardens, livestock rearing) have increased dietary diversity. ${ }^{22}$ Inclusion of behaviour change and women's empowerment interventions, in particular, has been key to enhancing the impact of agriculture on nutrition outcomes. ${ }^{22-24}$

Digital Green-a global development organisationhas developed an agricultural intervention involving community-led production and dissemination of videos in community groups. This has resulted in large improvements in agricultural practices and agricultural productivity in Bihar, India. ${ }^{25}$ During an earlier study by the Strengthening Partnerships, Results and Innovations Globally (SPRING) project, we found it was feasible to integrate content on maternal and child nutrition into 


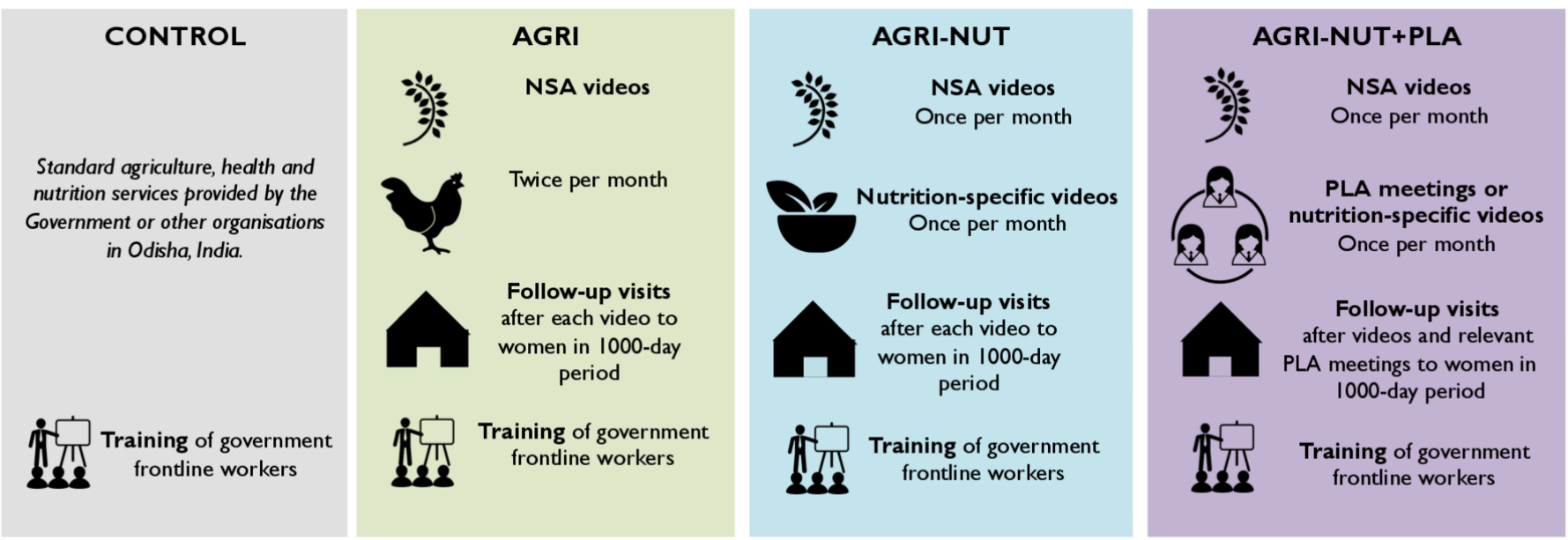

Figure 1 Overview of Upscaling Participatory Videos and Action for Agriculture and Nutrition (UPAVAN) interventions, taken from Kadiyala et al. ${ }^{20} \mathrm{NSA}$, nutrition-sensitive agriculture; PLA, Participatory Learning and Action.

similar agricultural videos. ${ }^{26}$ UPAVAN tests whether three 'nutrition-sensitive' agriculture (NSA) variants of this approach (figure 1) can improve maternal and child nutrition in Odisha, India.

The three intervention variants are:

1. Fortnightly women's groups viewing and discussing videos on NSA practices, and home visits to encourage the adoption of new practices shown in videos.

2. Fortnightly women's groups viewing and discussing videos on NSA, and 'nutrition-specific' behaviours (without agriculture content), plus home visits.

3. Fortnightly women's groups viewing and discussing videos on NSA and nutrition-specific behaviours, combined with a cycle of PLA group meetings, plus home visits. With help from the videos, these PLA meetings encouraged members to collectively understand the problem of undernutrition, and then identify, prioritise and act on locally feasible solutions to address this problem.

Local VARRAT staff facilitated the video disseminations, PLA meetings and home visits, and the interventions were open to all women in the community, although men were not discouraged from observing the video disseminations and PLA meetings. All interventions began with community mobilisation activities, and training of programme staff on maternal and child nutrition, hygiene and NSA.

Each intervention is compared with a control arm receiving standard government services and a 2-day nutrition training to government female community health workers, provided in all arms. The interventions began in April 2017 and ended in November 2019.

\section{Unpacking possible behaviours to prioritise}

The UPAVAN trial provides an apt example of the multiple pathways, behaviours and associated capabilities, opportunities and motivations that could change a single health outcome, as illustrated in figure 2.

There are several ways that NSA could improve nutrition outcomes (left-hand box; figure 2). Within a pathway there are many crops or agricultural behaviours that we could promote (middle box; figure 2), and people may have various capabilities, opportunities or motivations that encourage or improve behaviour change (righthand box; figure 2).

\section{Stages of intervention development}

Figure 3 shows the key stages by which we prioritised which topics to address in UPAVAN's videos, PLA meetings and home visits.

First, we hypothesised which NSA and nutrition-specific pathways were most likely to improve our outcomes by collectively developing a definition of "nutrition-sensitive agriculture', and a Theory of Change that outlined possible impact pathways. Next, we prioritised specific behaviours within these pathways. This was informed by published evidence and formative research. ${ }^{27}$ An operational protocol of implementation processes kept the team focused on the prioritised pathways and behaviours. Finally, from the prioritised pathways (in the Theory of Change) and associated prioritised NSA and nutritionspecific behaviours (from the formative research), we identified capabilities, opportunities and motivations to be addressed in each video or PLA meeting and associated home visit.

\section{PRIORITISING IMPACT PATHWAYS}

To decide on our priority pathways, we used the following inputs: conceptual frameworks, published empirical evidence, a feasibility study, formative research and local knowledge from VARRAT, Digital Green and Ekjut team members.

\section{Existing conceptual frameworks}

Several conceptual frameworks on the links between agriculture and nutrition already exist. ${ }^{28-30}$ We grounded our working definition of 'nutrition-sensitive agriculture' and our Theory of Change in these frameworks, and only included pathways and practices relevant for our study. Using Kadiyala et $a l^{28}$ we ignored macro-level factors 
Pathways to impact

\section{Behaviour}

Possible capabilities, opportunities and motivations to address

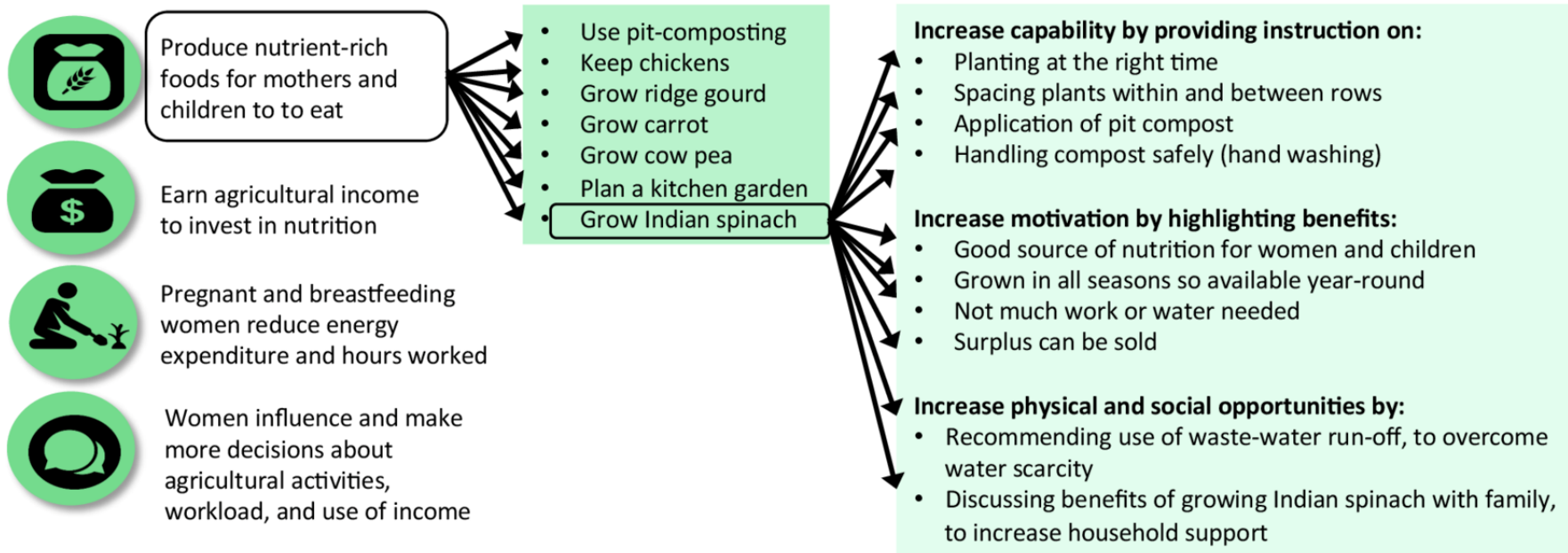

Contextual factors: Rain-fed agriculture, water scarcity, low labour and land productivity, gender roles, seasonal migration, weak supply chains, strong complementarity with government health and nutrition programmes

Figure 2 Unpacking the possible pathways, behaviours and capabilities, opportunities and motivations that Upscaling Participatory Videos and Action for Agriculture and Nutrition (UPAVAN) could prioritise.

such as domestic food trade, health infrastructure and employment, which would not realistically be influenced by our intervention. Instead, we focused on household and intra-household-level factors, such as income, agricultural production, food expenditures, caring capacity and practices, and women's empowerment and energy expenditure.

\section{Existing empirical evidence}

In particular, we drew from nutrition interventions in India, ${ }^{5} \mathrm{Nepal}^{731}$ and Bangladesh, ${ }^{9}$ and homestead gardening interventions. ${ }^{32} 33$ Using existing evidence, we deprioritised two possible pathways on hygiene and care-seeking during illness. Although infection intuitively seems like an important factor to address and is an important determinant of nutritional status, ${ }^{34} 35$ a PLA group intervention in India improved hand washing practices but had no effect on child illness, ${ }^{5}$ and another trial found no effects of a water, sanitation and hygiene intervention on child length, ${ }^{36}$ perhaps due to multiple exposures to infection risks. Therefore, we chose not to focus on infection reduction, but did emphasise the importance of hand washing as a preventative measure, in relation to promoted agricultural practices, such as compost making and chicken farming. ${ }^{37}$ The PLA intervention also found that seeking care from a nurse or doctor was not amenable to change, ${ }^{5}$ and we were constrained by supply-side limitations in the quality of care, so we also deprioritised this pathway.

\section{Feasibility study and formative research}

We conducted a feasibility study ${ }^{38}$ and formative research $^{27} 39$ before starting intervention activities. The formative research was led by technical experts, JSI
Research and Training Institute, in collaboration with implementing teams (VARRAT, Digital Green and Ekjut), and aimed to (1) understand community members' perspectives; (2) develop a list of foods, crops and livestock; (3) identify barriers and enablers to changing agriculture and nutrition behaviours, including stakeholders involved; and (4) create a seasonal calendar of agricultural processes, cash flows, labour and gender roles. Methods used were focus group discussions, a participatory food ranking using pile sorts, an exercise to fill out daily activity charts for participants and their family members, and direct observation via transect walks through selected villages.

This provided contextual information on which pathways might be relevant and amenable to change. For example, daily activity charts created by women, their husbands and mothers-in-law showed that a major issue was the heavy work burdens that women of reproductive age faced, compared with men and older women. Focus group discussions indicated that it could be feasible to reduce women's workload, and heavy or time-consuming tasks could be shifted to other adults in the household.

\section{Local knowledge from the implementation team}

We also drew from the local team's knowledge about agricultural and nutrition practices and barriers to change. For example, we did not prioritise improving value-chain pathways (eg, improving cold storage facilities for agriculture produce), because the team considered it unfeasible to change in the time frame. Instead, we focused on increasing agricultural income, since agriculture is the main livelihood, ${ }^{40}$ and income is an important constraint to agricultural productivity and dietary adequacy. 


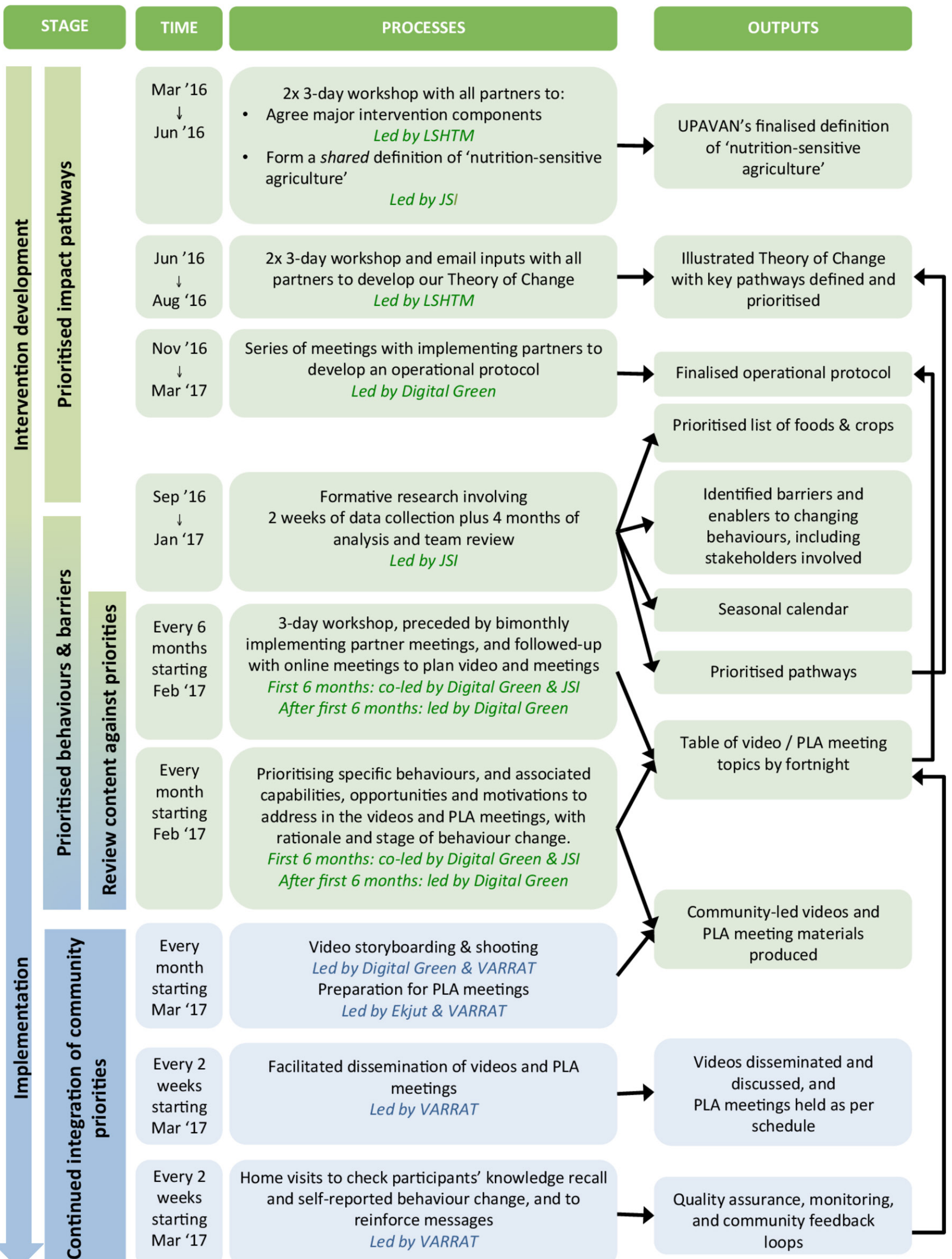

Figure 3 Key stages in the development and prioritisation of Upscaling Participatory Videos and Action for Agriculture and Nutrition (UPAVAN) interventions. PLA, Participatory Learning and Action; VARRAT, Voluntary Association for Rural Reconstruction and Appropriate Technology. 
This left us with four prioritised NSA pathways (in figure 2) and two nutrition-specific pathways (improving maternal diets and improving child feeding practices). This Theory of Change, and methods of measuring these pathways, are given in our protocol. ${ }^{20}$

\section{PRIORITISING BEHAVIOURS AND BARRIERS}

After prioritising our key pathways, we needed a second round of prioritisation to select specific behaviours to address. We initially drew on our formative research, local knowledge and impact pathways.

\section{Formative research and local knowledge}

To prioritise the NSA behaviours, we overlaid the seasonal calendar with the crop and food list, and prioritised foods and crops based on:

- Time of year.

- Nutritive value.

- Economic value.

- Labour requirements.

- Cost and accessibility of inputs required.

- Feasibility of adoption.

For example, based on the seasonal calendar and local agronomic feasibility, videos on cultivating Indian spinach with wastewater and locally available seeds were disseminated during lean seasons when water is scarce.

To identify which nutrition-sensitive agricultural behaviours to promote within a prioritised food or crop, we identified what single, feasible change was most important. Similar to Berti et $a l_{,}^{41}$ the local team determined whether the food or crop was new to the area but could be promoted (such as carrots), or whether it was already produced but practices could be improved (eg, improving spacing of Indian spinach, or encouraging people to eat pumpkins that they already grow).

We then filled gaps in the video and PLA meeting calendar with behaviours that were less time-sensitive but nevertheless important, such as videos explaining the concept of NSA, or household budgeting.

For each behaviour, we identified reasons why people were not already doing the practice. For example, our formative research found a belief in some communities that Indian spinach is harmful for children and pregnant women, often imposed by mothers-in-law. The videos therefore featured a story of a mothers-in-law's journey of 'pre-contemplation' to 'action', during which she became an advocate for the consumption of Indian spinach.

The team also identified ways to address multiple pathways concurrently. For example, a series of videos focused on producing crops that require minimal labour and are of economically high value, such as mushrooms. Growing mushrooms could supplement income and reduce women's energy expenditure.

Recognising that the prevalence, appropriateness, and feasibility of nutrition and agricultural practices would vary by multiple factors (such as geography, caste, wealth and season), we tried to capture a breadth of perspectives in the formative research. When facilitating the group discussions, meeting content was tailored to be specific to the participants. Group members discussed barriers and solutions to adoption that may be more or less relevant for them (eg, water scarcity, restrictive gender roles) and shared their experiences with each other. The PLA meetings are specifically designed to be locally appropriate because groups themselves identify and prioritise salient problems, solutions and strategies to implement.

\section{Reviewing intervention content in relation to our priorities}

We reviewed the video content and group meeting plans every 6 months, and assessed whether we were giving each pathway equal weight. We found some pathways easier to address than others. Notably, the promotion of nutrientrich foods for household consumption was conceptually simple, whereas increasing women's decision-making power in the household proved difficult to conceptualise, storyboard and film.

We also mapped each specific promoted behaviour against the transtheoretical model of behaviour change, ${ }^{13}$ based on whether the behaviour was generally (1) new to the community ('pre-contemplation'); (2) being considered ('contemplation'); (3) of interest to the community ('preparation'), (4) being first adopted ('action'); (5) being continued ('maintenance'); or (6) being modelled to others in the community ('termination').

Table 1 illustrates this mapping using a series of videos on chicken farming.

This mapping exercise allowed us to be systematic in ensuring balance across pathways and track behaviour change stages, and kept us focused on a confined, core set of behaviours.

\section{Continued integration of community priorities}

Once the intervention was underway, we drew heavily on community feedback (figure 4). To balance expert opinion, evidence and community priorities, we often arrived at decisions through consultation with the group meeting facilitators.

To create the videos, we developed a storyboard based on our agreed behaviours and barriers, written in local Odia language, and then filmed with community members. We collected feedback from facilitators, local government health workers (Anganwadi workers and agricultural extension workers) and protagonists. After showing the videos, facilitators discussed with participants the appropriateness of the videos, reasons why they may not adopt promoted behaviours and ideas of new topics, all of which informed future videos.

Unique to the PLA meetings, an additional level of prioritisation occurred in each women's group. We used a list of nutrition-specific behaviours identified from the formative work and local knowledge to create picture cards for participants to collectively discuss, prioritise and find strategies to implement. This meant that, in this intervention component, PLA groups would discuss a common set of topics related to maternal and child 
Table 1 An example of mapping videos, with specific behaviours, capabilities, opportunities and motivations addressed, to the main pathway and transtheoretical behaviour change stage

\begin{tabular}{|c|c|c|c|c|}
\hline Title of the video & $\begin{array}{l}\text { Main prioritised } \\
\text { pathway }\end{array}$ & Specific behaviours promoted & $\begin{array}{l}\text { Capabilities, opportunities and } \\
\text { motivations addressed }\end{array}$ & $\begin{array}{l}\text { Transtheoretical } \\
\text { behaviour change } \\
\text { stage }\end{array}$ \\
\hline $\begin{array}{l}\text { Benefits of chicken } \\
\text { farming }\end{array}$ & Produce food & $\begin{array}{l}\text { Raise chickens } \\
\text { Pregnant women and children eat } \\
\text { the meat and eggs produced }\end{array}$ & $\begin{array}{l}\text { Motivate participants to raise } \\
\text { chickens by highlighting benefits } \\
\text { (source of income and nutritious } \\
\text { food) }\end{array}$ & Contemplation \\
\hline $\begin{array}{l}\text { How to practise } \\
\text { improved chicken } \\
\text { farming }\end{array}$ & Earn income & $\begin{array}{l}\text { Keep chickens in a small house, } \\
\text { especially at night, to keep them } \\
\text { safe and so they lay more eggs } \\
\text { Families decide together who } \\
\text { should care for the chickens and } \\
\text { what to do with the produce and } \\
\text { income from surplus }\end{array}$ & $\begin{array}{l}\text { Increase capabilities to improve } \\
\text { chicken farming by providing } \\
\text { instruction on penning at night } \\
\text { Increase women's social } \\
\text { opportunities to be involved in } \\
\text { decisions about workload and use } \\
\text { of chicken produce and income by } \\
\text { promoting joint decision-making }\end{array}$ & Preparation \\
\hline $\begin{array}{l}\text { Benefits of chicken } \\
\text { farming - Testimonial }\end{array}$ & Produce food & $\begin{array}{l}\text { Regularly immunise chickens to } \\
\text { ensure high survival rates } \\
\text { Pregnant and breastfeeding women, } \\
\text { and children aged 6-24 months } \\
\text { should consume eggs at least every } \\
\text { other day } \\
\text { Sell surplus chicks, chickens and } \\
\text { eggs only after there is enough for } \\
\text { women and children to consume } \\
\text { eggs at least every other day }\end{array}$ & $\begin{array}{l}\text { Motivate participants to keep } \\
\text { chickens and immunise them by } \\
\text { sharing a success story } \\
\text { Increase capability to keep } \\
\text { healthy chickens and improve } \\
\text { diets by providing instruction } \\
\text { on immunisation and egg } \\
\text { consumption }\end{array}$ & Action \\
\hline $\begin{array}{l}\text { How to practise } \\
\text { improved chicken } \\
\text { farming }\end{array}$ & Produce food & $\begin{array}{l}\text { Keep chickens in a small house, } \\
\text { especially at night, to keep them } \\
\text { safe and so they lay more eggs } \\
\text { Families should decide together } \\
\text { who should care for the chickens } \\
\text { and what to do with the produce }\end{array}$ & $\begin{array}{l}\text { Increase capability to improve } \\
\text { chicken farming by reinforcing } \\
\text { instruction on penning } \\
\text { Increase women's opportunities } \\
\text { to be involved in decisions about } \\
\text { workload and use of chicken } \\
\text { produce }\end{array}$ & Maintenance \\
\hline
\end{tabular}

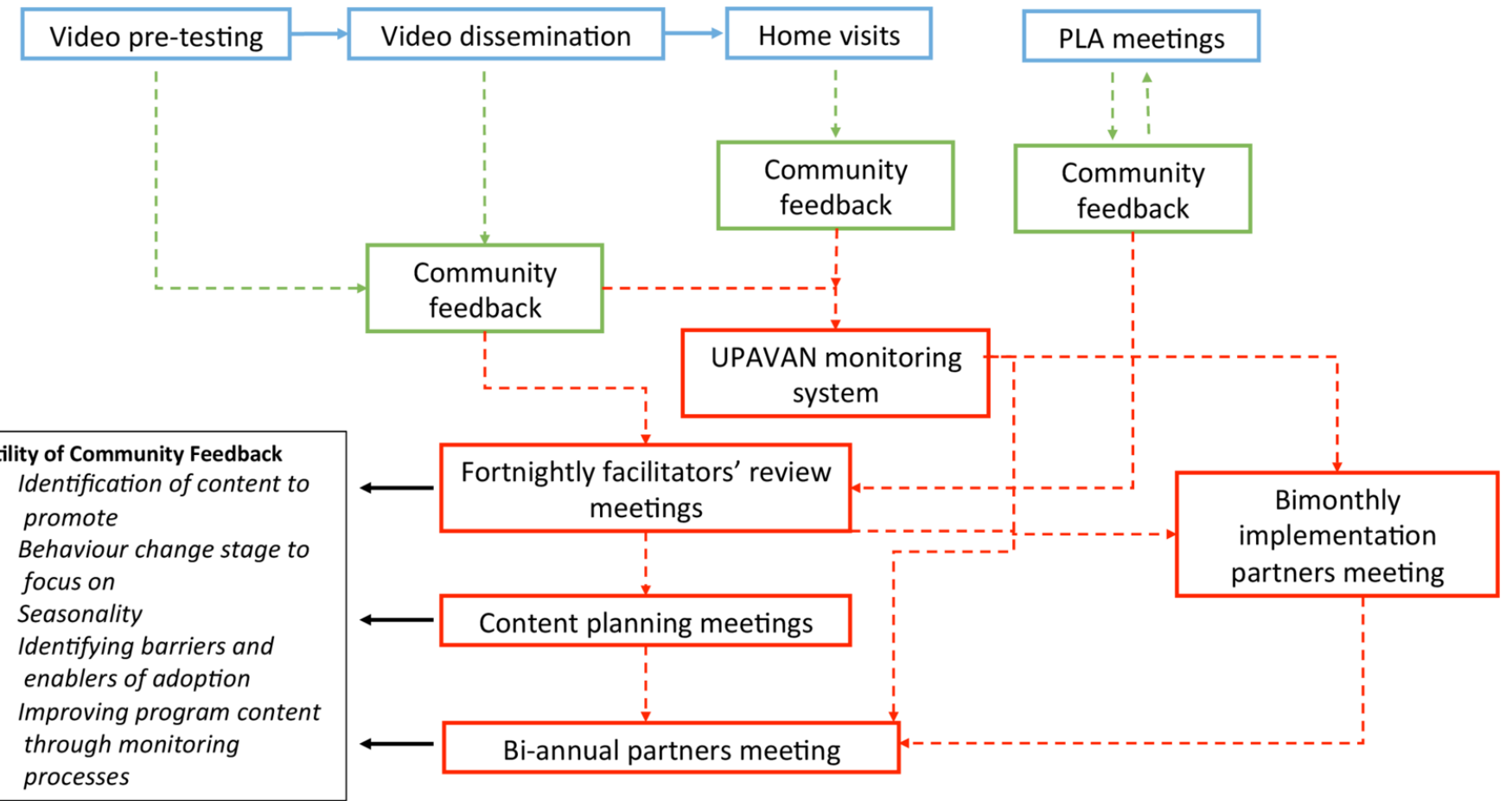

Figure 4 Flow of community feedback in Upscaling Participatory Videos and Action for Agriculture and Nutrition (UPAVAN). PLA, Participatory Learning and Action. 
undernutrition, but each group differently prioritised which problems they wanted to address and strategies to implement.

Unlike in the group meetings where women may feel shy to speak up, home visits gave an opportunity for participants to quietly discuss the relevance of, and enablers and barriers to, promoted behaviours. During the home visits, facilitators also collected data on participants' recall of the messages shown in videos or discussed in meetings, and their adoption of promoted behaviours.

This feedback was collated by the VARRAT team during fortnightly review meetings with the group facilitators, and used to plan content and review progress. The quantitative data collected at the home visits were analysed by Digital Green to quantify coverage gaps, knowledge recall and behaviour adoption.

Based on feedback from participants and group facilitators, new topics were introduced and popular topics were repeated. For instance, we did not plan to promote behaviours on limiting unhealthy snacks, but qualitative feedback identified unhealthy snacks displacing nutrientrich foods as a concern. Therefore, we included this as a new topic in videos and PLA meetings. A popular topic that we repeated was Indian spinach. Monitoring data from the home visits found that it was popular: 1344 (11.5\% of video viewers) households adopted it after the first video, and qualitative feedback also showed community demand for another video. Therefore, a second video was developed to reinforce the benefits of growing Indian spinach with improved cultivation practices. Monitoring data showed that adoption more than doubled to 2932 $(15.2 \%)$ households.

\section{REFLECTIONS ON LESSONS LEARNT}

We reflect on four key lessons learnt from our experience of prioritising behaviours and barriers to address in a complex behaviour change intervention.

First, our shared understanding of complex concepts such as 'nutrition-sensitive agriculture', 'women's empowerment' and pathways to impact across all partners enabled group facilitators to understand and clearly communicate these concepts. The consensus-based approach in developing the Theory of Change enabled mutual knowledge exchange between partners and was instrumental in subsequently structuring the video disseminations and PLA meeting plans. Our Theory of Change was the guiding framework for designing, prioritising and communicating complex behaviours.

Second, there is a delicate trade-off between time taken to design the intervention and time taken to reach consensus across partners. We spent around 1 year on the set-up of this intervention, which included formative research. Time-consuming factors were the complexity and number interventions (as this was a four-arm trial), our consensus-based approach and, relatedly, UPAVAN's large number of partner organisations. However, we think this investment in set-up resulted in efficiencies later on, due to less reliance on international technical expert inputs, smooth intervention delivery and equitable partnerships. There were also factors that ensured we used this set-up time efficiently. Our interventions built on pre-existing models of implementation (Digital Green's video approach and PLA), we had already conducted a feasibility study, and the implementing partners had extensive local knowledge.

Third, there is a related balance between using inputs from technical experts while staying true to the community-led, participatory principles inbuilt in Digital Green's video-making and in PLA. In UPAVAN, key decisions about intervention design that we discussed at length, but we feel were worth the time, regarded the intervention dosage (number of meetings per month and number of groups per population) and prioritising which pathways to focus on. Since complex programmes often involve programmatic trade-offs, and ideological or epistemological differences among programme partners, reaching consensus was important and empowering to all partners involved.

Finally, for such complex interventions to work, fidelity to the implementation design is essential. Continuous review with a strong monitoring system-that integrated community feedback, mapped the Theory of Change and behaviour change stages, and assessed progress against agreed targets-was both essential and doable. With time, local implementers were able to lead this review in a self-reflective process.

\section{CONCLUSION}

This article documented the process through which the team leading a complex NSA trial (UPAVAN) selected key pathways to impact, and identified which behaviours to promote and barriers to address. Key inputs to these processes were existing conceptual frameworks and behaviour change models, empirical evidence, feasibility and formative research, a collectively agreed on Theory of Change, local knowledge of implementing partners, and community feedback. We found the most useful inputs were the shared understanding of impact pathways and strong community feedback loops.

Impact and process evaluations will reveal whether these approaches to prioritise behaviours and design a behaviour change intervention are effective for improving maternal and child nutrition, and the pathways by which the interventions did or did not work.

\section{Author affiliations}

${ }^{1}$ Department of Population Health, London School of Hygiene and Tropical Medicine, London, UK

${ }^{2}$ Friedman School of Nutrition Science and Policy, Tufts University, Medford,

Massachusetts, USA

${ }^{3}$ Digital Green, Bhubaneswar, India

${ }^{4}$ Ekjut, Chakradharpur, India

${ }^{5}$ John Snow Inc, Boston, Massachusetts, USA

${ }^{6}$ VARRAT, Keonjhar, India

${ }^{7}$ University College London Institute of Child Health, London, UK 
Contributors $\mathrm{HH}-\mathrm{F}$ and SKa formulated the paper idea and structure. HH-F wrote the manuscript with substantial contributions from all authors. All authors made substantial contributions to the intervention development. All authors read and approved the final manuscript.

Funding This research was funded by Bill \& Melinda Gates Foundation and UK Government's Department for International Development (award number OPP1136656). Additional funding to support participation in implementation design, the design and execution of formative research, the development of training, and participation in partner review meetings was provided by USAID through the SPRING nutrition project (Cooperative Agreements AID-386-A-15-00008 and AID-OAA-A-11-00031). Funding of author HH-F during the write-up period was provided by a Sir Henry Wellcome Fellowship (grant 210894/Z/18/Z). The funders had no role in the research.

Competing interests None declared.

Patient consent for publication Not required.

Provenance and peer review Not commissioned; externally peer reviewed.

Data availability statement There are no data in this work.

Open access This is an open access article distributed in accordance with the Creative Commons Attribution 4.0 Unported (CC BY 4.0) license, which permits others to copy, redistribute, remix, transform and build upon this work for any purpose, provided the original work is properly cited, a link to the licence is given, and indication of whether changes were made. See: https://creativecommons.org/ licenses/by/4.0/.

\section{ORCID iD}

Helen Harris-Fry http://orcid.org/0000-0003-2367-908X

\section{REFERENCES}

1 Briscoe C, Aboud F. Behaviour change communication targeting four health behaviours in developing countries: a review of change techniques. Soc Sci Med 2012;75:612-21.

2 Hardeman W, Johnston M, Johnston D, et al. Application of the theory of planned behaviour in behaviour change interventions: a systematic review. Psychol Health 2002;17:123-58.

3 Girard AW, Olude O. Nutrition education and counselling provided during pregnancy: effects on maternal, neonatal and child health outcomes. Paediatr Perinat Epidemiol 2012;26:191-204.

4 Spahn JM, Reeves RS, Keim KS, et al. State of the evidence regarding behavior change theories and strategies in nutrition counseling to facilitate health and food behavior change. J Am Diet Assoc 2010;110:879-91.

5 Nair N, Tripathy P, Sachdev HS, et al. Effect of participatory women's groups and counselling through home visits on children's linear growth in rural eastern India (caring trial): a cluster-randomised controlled trial. Lancet Glob Health 2017;5:e1004-16.

6 Biran A, Schmidt W-P, Varadharajan KS, et al. Effect of a behaviour-change intervention on handwashing with soap in India (SuperAmma): a cluster-randomised trial. Lancet Glob Health 2014;2:e145-54.

7 Saville NM, Shrestha BP, Style S, et al. Impact on birth weight and child growth of participatory learning and action women's groups with and without transfers of food or cash during pregnancy: findings of the low birth weight South Asia cluster-randomised controlled trial (LBWSAT) in Nepal. PLoS One 2018;13:e0194064

8 Sarrassat S, Meda N, Badolo $\mathrm{H}$, et al. Effect of a mass radio campaign on family behaviours and child survival in Burkina Faso: a repeated cross-sectional, cluster-randomised trial. Lancet Glob Health 2018;6:e330-41.

9 Menon P, Nguyen PH, Saha KK, et al. Impacts on breastfeeding practices of at-scale strategies that combine intensive interpersonal counseling, mass media, and community mobilization: results of cluster-randomized program evaluations in Bangladesh and Viet Nam. PLoS Med 2016;13:e1002159.

10 Fottrell E, Ahmed N, Morrison J, et al. Community groups or mobile phone messaging to prevent and control type 2 diabetes and intermediate hyperglycaemia in Bangladesh (DMagic): a cluster-randomised controlled trial. Lancet Diabetes Endocrinol 2019;7:200-12.

11 Black RE, Victora CG, Walker SP, et al. Maternal and child undernutrition and overweight in low-income and middle-income countries. Lancet 2013;382:427-51.
12 Michie S, van Stralen MM, West R. The behaviour change wheel: a new method for characterising and designing behaviour change interventions. Implement Sci 2011;6:42

13 Prochaska JO, DiClemente CC. Transtheoretical therapy: toward a more integrative model of change. Psychotherapy: Theory, Research \& Practice 1982;19:276-88.

14 Bawden D, Robinson L. The dark side of information: overload, anxiety and other paradoxes and pathologies. J Inf Sci 2009;35:180-91.

15 Botti S, lyengar SS. The dark side of choice: when choice impairs social welfare. Journal of Public Policy \& Marketing 2006;25:24-38.

16 Kim SS, Nguyen PH, Tran LM, et al. Different combination of behavior change interventions and frequency of interpersonal contacts are associated with infant and young child feeding practices in Bangladesh, Ethiopia, and Viet Nam. Current Developments in Nutrition 2019.

17 Fottrell E, Azad K, Kuddus A, et al. The effect of increased coverage of participatory women's groups on neonatal mortality in Bangladesh: a cluster randomized trial. JAMA Pediatr 2013;167:816-25.

18 Prost A, Colbourn T, Seward N, et al. Women's groups practising participatory learning and action to improve maternal and newborn health in low-resource settings: a systematic review and metaanalysis. Lancet 2013;381:1736-46.

19 Diressie MT, Smale M, Leclair M, et al. On the radio: using serial Mini-Dramas to promote orange sweet potato in Uganda agriculture nutrition health Academy. Accra, Ghana: ANH Academy, 2018.

20 Kadiyala S, Prost A, Harris-Fry H, et al. Upscaling participatory action and Videos for agriculture and nutrition (UPAVAN) trial comparing three variants of a nutrition-sensitive agricultural extension intervention to improve maternal and child nutritional outcomes in rural Odisha, India: study protocol for a cluster randomised controlled trial. Trials 2018;19:176.

21 NFHS-4. National family health survey, India. in: (IIPS) IIfPS, ED. Mumbai 2016.

22 Berti PR, Krasevec J, FitzGerald S. A review of the effectiveness of agriculture interventions in improving nutrition outcomes. Public Health Nutr 2004;7:599-609.

23 Ruel MT, Quisumbing AR, Balagamwala M. Nutrition-sensitive agriculture: what have we learned so far? Global Food Security 2018;17:128-53.

24 Webb P, Kennedy E. Impacts of agriculture on nutrition: nature of the evidence and research gaps. Food Nutr Bull 2014;35:126-32.

25 Toyama K. Digital green RCT: final report with preliminary analysis. Jameel Poverty Action Lab South Asia 2017.

26 Kadiyala S, Roopnaraine T, Margolies A, et al. Using a communityled video approach to promote maternal infant and young child nutrition in Odisha India: results from a pilot and feasibility study, 2014.

27 Aakesson A, Cunningham S, Danton $\mathrm{H}$, et al. Using farming families' perspectives to inform recommended priority practices: UPAVAN Formative Research Report. Arlington, VA.: Strengthening Partnerships, Results, and Innovations in Nutrition Globally (SPRING) project, 2017.

28 Kadiyala S, Harris J, Headey D, et al. Agriculture and nutrition in India: mapping evidence to pathways. Ann N Y Acad Sci 2014;1331:43-56.

29 Haddad L. A conceptual framework for assessing agriculturenutrition linkages. Food Nutr Bull 2000;21:367-73.

30 Kanter R, Walls HL, Tak M, et al. A conceptual framework for understanding the impacts of agriculture and food system policies on nutrition and health. Food Security 2015;7:767-77.

31 Morrison J, Dulal S, Harris-Fry $\mathrm{H}$, et al. Formative qualitative research to develop community-based interventions addressing low birth weight in the plains of Nepal. Public Health Nutr 2018;21:377-84

32 Bushamuka VN, de Pee S, Talukder A, et al. Impact of a Homestead gardening program on household food security and empowerment of women in Bangladesh. Food Nutr Bull 2005;26:17-25.

33 Talukder A, Kiess L, Huq N, et al. Increasing the production and consumption of vitamin A-rich fruits and vegetables: lessons learned in taking the Bangladesh Homestead gardening programme to a national scale. Food Nutr Bull 2000;21:165-72.

34 Guerrant RL, Schorling JB, McAuliffe JF, et al. Diarrhea as a cause and an effect of malnutrition: diarrhea prevents catch-up growth and malnutrition increases diarrhea frequency and duration. Am J Trop Med Hyg 1992;47:28-35.

35 Drakesmith $\mathrm{H}$, Prentice AM. Hepcidin and the iron-infection axis. Science 2012;338:768-72.

36 Prendergast AJ, Chasekwa B, Evans C, et al. Independent and combined effects of improved water, sanitation, and hygiene, and 
improved complementary feeding, on stunting and anaemia among HIV-exposed children in rural Zimbabwe: a cluster-randomised controlled trial. Lancet Child Adolesc Health 2019;3:77-90.

37 Gelli A, Headey D, Becquey E, et al. Poultry husbandry, water, sanitation, and hygiene practices, and child anthropometry in rural Burkina Faso. Matern Child Nutr 2019;15:e12818.

38 Kadiyala S, Morgan EH, Cyriac S, et al. Adapting agriculture platforms for nutrition: a case study of a participatory, video-based agricultural extension platform in India. PLoS One 2016;11:e0164002.

39 SPRING. SPRING/Digital green collaboration and feasibility study: a report on the key findings of the formative research conducted at the initiation of the project in 2012. Arlington, Virginia, USA: Strengthening Partnerships, Results, and Innovations in Nutrition Globally (SPRING) project, 2016.

40 Government of Odisha. Status of Agriculture in Odisha 2014 15. In: Department of agriculture and farmers' Empowerment. Bhubaneswar, India, 2014.

41 Berti PR, Desrochers RE, Van HP, et al. The process of developing a nutrition-sensitive agriculture intervention: a multi-site experience. Food Security 2016;8:1053-68. 